\title{
Offloading Decision Algorithm Based on Distance Weighted K- Nearest Neighbor in Power Internet of Things
}

\author{
Jun Jia(1), Jiangtao Xu $\mathbf{u}^{\left(1^{*}\right)}$, Wenqing Cui(2), Fengbo Tao(1), Guojiang Zhang ${ }^{(3)}$, Chengbo \\ Hu(1), Zhaohui Zhang(1), Ziquan Liu(1)
}

(1) State Grid Jiangsu Electric Power Co. LTD. Research Institute, Nanjing 211103, CHINA e-mail:136933974@qq.com

(2) School of Information Science and Engineering, Southeast University, Nanjing 210096, CHINA

(3) State Grid Jiangsu Electric Power Co. LTD., Nanjing 210000, CHINA

\section{SUMMARY}

With the widespread popularity of power Internet of Things (PIoT), the data collected from smart meters are growing explosively, which makes the calculation task of power data more and more complex. In order to improve computing power and maximize resource utilization, an offloading decision algorithm based on weighted K-nearest neighbor (WKNN) is proposed. It first collects the training set required by the WKNN-based algorithm, including the Received Signal Strength (RSS) required for offloading, the transmission rate, and the load balance of the Access Point (AP), and then the Euclidean distance between the training set and the sample is weighted by Gaussian function. Finally, the result with the largest $K$ similarities in the training set is the offloading result. The simulation results show that the proposed algorithm reduces the offloading delay of the computing tasks and improves the resource utilization rate effectively when the number of meters increases in the network, which ensures that the resources of the mobile edge computing (MEC) servers in the system can be effectively and evenly utilized.

KEY WORDS: Power Internet of Things (PIoT); offloading decision; weighted K-nearest neighbor (WKNN).

\section{INTRODUCTION}

The Internet of Things (IoT) technology and system used in smart grids are called power Internet of Things (PIoT) [1-2]. With the increasing popularity of PIoT in smart grids, the data and information collected from smart meters are experiencing explosive growth, the computing tasks between power data centers are becoming more and more complex, and the quality of service (QoS) requirements is also becoming higher. Under this background, the key 
issue for supporting the development and scheduling strategy of smart grids is how to realize the efficient transmission and scheduling of IoT data in the PIoT data center.

In recent years, cloud computing has become a recognized next-generation computing infrastructure by both academia and industry. It offers many features such as scalability, agility, and economic efficiency compared to traditional infrastructure. Since cloud computing is essentially a computing platform for massive data processing, it can be applied to the processing of massive computing tasks generated in the PIoT for improving its operational efficiency and resource utilization. However, with the increasing demand for low delay and computing of smart meters in the PIoT, smart devices cannot meet the size, energy, and other constraints. Therefore, the edge cloud server installed near the smart meter is introduced into cloud computing. This technology is called Mobile Edge Computing (MEC) [3], which facilitates the smart meter to offload to the near-end MEC server and reduce task offloading costs with lower time delay constraints.

Some research scholars have conducted related research around the computational offloading process over the years. In the mobile edge cloud computing system, a privacy protection offloading algorithm based on the Constrained Markov Decision Process (CMDP) in the MEC system was proposed in the literature [4]. It enabled mobile users to achieve minimum delay and energy consumption while guaranteeing a pre-set privacy level. However, the convergence time of the algorithm was long, and the given MEC scenarios were limited. The algorithm did not consider the more general multi-state wireless channel and multi-task processing scenarios. In order to solve the problem of unbalanced computing load in the network space, it is necessary to avoid large delays by parallel offloading between small base stations (SBS). The literature [5] proposed a new online SBS parallel offloading framework OPEN, which uses the Lyapunov method to optimize the performance of edge computing under limited energy budget that a single SBS can withstand without future information. The results showed that the OPEN algorithm could minimize system delay and increase system energy utilization without taking into account the transmission costs and resource benefits caused by the offloading process. When the number of users in the system increases, the literature [6] puts forward a new partial compression offloading model that can improve users' QoS and reduce the offloading delay by minimizing the weighted sum of the delay of all devices. Compared to traditional local offloading and cloud offloading models, the partial compression offloading model effectively reduces the end-to-end delay. In order to minimize the cost of offloading, the literature [7] proposed a Markov Decision Process (MDP) method to enable mobile users to make the best offloading decision in a specific mobile cloud. Taking into account the impact of cloudlet distance, mobility and time-varying channel characteristics on the success of the offloading action, the proposed MDP method obtained the best offloading strategy to determine the number of tasks mobile user processes locally and offloads them to each nearby mobile cloud maximizing the overall utility of the user and minimizing energy consumption and offloading costs. The literature [8] presented a multi-user task offload scheduling algorithm that guarantees the performance of MEC services. This algorithm uses the KKT condition to solve the optimization problem of energy minimization in the MEC system, and the mobile user decides whether to perform task offloading according to the energy consumption and completion performance of the MEC server. Especially when the size of the offloading task becomes larger, the offloading scheme proposed in the literature [9] is superior to the local offloading and full offloading scheme, which reduces the mobile energy consumption and improves the performance of the MEC server. The literature [10] studied the cooperation problem of multiple MEC servers and base stations in the MEC environment. It 
enhances the computational offloading service of MEC-BS by offloading additional tasks to the MEC-BS connected to it. The algorithm proposed in this document effectively solves the problem of maximizing the time and energy consumption for mobile devices. However, it didn't consider the load condition of the base station which easily causes overload and other problems affecting the QoS of the mobile device. In addition, some research scholars obtained results for edge cloud computing technology in the PIoT. The literature [11] proposed an edge computing framework for real-time monitoring, which transferred computing from the cloud computing center to the remote device server, and the deep learning algorithm was run in the edge server in order to reduce the delay and improve the frame rate. Furthermore, this literature also used a simulated annealing strategy to solve the scheduling problem of the edge detection system. In the [12], in view of the needs of emergency communication with electric off-network, a multi-hop relay direct communication method for edge networks was raised to ensure the self-organized communication without core network in an emergency. At the same time, it also proposed an intelligent routing algorithm based on the smoothness and obtained the calculation and update of the smoothness through the edge calculation method. However, it only applied the edge computing to the off-network emergency communication of the PioT, at a low utilization rate, which is difficult to be popularized. In the literature [13], user realtime electricity consumption information is offloaded at edge nodes in the power supply and demand system, which alleviates the burden of enterprises to collect electricity information and ensures the orderly power supply according to the importance of power events in the case of power shortage. However, the details of the offloading process are not described in the literature.

The above literature proved that although the offloading algorithm in MEC system achieved excellent research results for improving resource utilization, these algorithm cannot be directly used in the MEC-based PIoT system, and specific designs should be addressed to satisfy the requirements of the smart meters with massive computing tasks in MEC-based PIoT. Therefore, this paper proposes an offloading decision algorithm based on Distance Weighted K-Nearest Neighbor (WKNN) for the MEC-based PIoT system, and the main contributions are as follows.

- The proposed algorithm is mainly divided into two parts: the acquisition and processing of the training set and the offloading decision based on the processed data set, using the WKNN method.

- The proposed algorithm comprehensively takes into account the received signal strength (RSS), transmission rate, and load balancing at the access point (AP) to make the offloading decision. The load includes the communication and computational resources load.

- Distance metric function and distance weighted function are defined to make local and different estimations for each new instance to be classified.

- The simulation results show that the performance of the proposed algorithm is significantly superior to the existing algorithms in terms of resource utilization rate and offloading delay.

The paper mainly consists of five sections as follows: Section 1 describes the PIoT and reviews the related researches on the offloading algorithm. Section 2 briefly introduces the architecture of the MEC-based power IoT system used in this paper, Section 3 elaborates on the distance weighted K-nearest neighbor (WKNN) algorithm, Section 4 presents simulation analysis and results, Section 5 gives the conclusions. 


\section{SYSTEM ARCHITECTURE}

In a MEC-based PIoT system, MEC servers are deployed around mobile users or smart meters. When multiple smart devices perform computational offloading, they need to allocate offloading connections reasonably to ensure maximum the utilization of computing and storage resources of each MEC server as well as to satisfy the load balancing of the network. The system architecture adopted in this paper includes smart meters, MEC servers, APs, and the main power network, as shown in Figure 1. It is a model that extends cloud computing and related services to the edge of the network. Since the main power network has a central cloud, the MEC server is connected to the main power network through a Wide Area Network (WAN) so as to process and transmit data and provide localized offloaded cloud services for smart meters. Through a wireless link, the smart meter is connected to the AP, and each AP is connected to one MEC server by a wired link, [14].

In this section, the smart meter $n(n \in N=\{1, \ldots, N\}$ ) connects to the surrounding access point resources $A P_{i}$ through the wireless channel. Given that there are $I$ access points around the smart meter $n$, then $i \in I=\{1, \ldots, I\}$. When the smart meter $n$ needs to perform a large number of computing tasks, limited by its own computing resources, it offloads the computing task to the MEC server connected to the AP and utilizes the strong computing power of the MEC server to improve the computing performance of the smart meter. When there are many APs and MEC servers around the smart meter, it is necessary to decide which AP and MEC servers should be used to offload the task so as to ensure that the resources of the MEC server are effectively and evenly utilized.

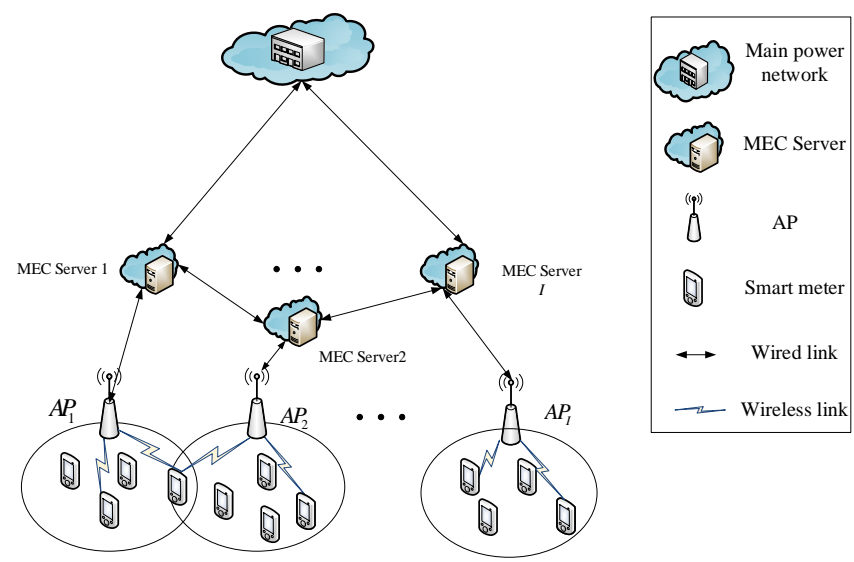

Fig. 1 MEC-based power loT system architecture

\section{OFFLOADING DECISION ALGORITHM BASED ON WKNN}

In order to solve the complex computing task of massive power data in PIoT, this paper proposes a WKNN-based offloading decision algorithm. This algorithm is mainly divided into two parts: the acquisition and processing of the training set and the offloading decision based on the processed data set, using the WKNN method. 


\subsection{DATA SET COLLECTION AND PROCESSING}

In this paper, the metrics such as the RSS, network transmission rate, and AP load balancing were used to make the offloading decision of the smart meter calculation tasks. The metrics collected by the MEC server were taken as a data set, which was processed by the WKNN algorithm to perform the offloading decision.

\section{A. Received Signal Strength}

The Received Signal Strength (RSS) reflects the channel quality of the $A P_{i}$ currently connected to the smart grid. It is expressed as:

$$
\operatorname{RSS}_{i}=K_{1}-K_{2} \log _{2}\left(d_{i}\right)+\mu(x)
$$

where, $K_{1}$ is the transmit power, $K_{2}$ is the path loss, $d_{i}$ is the distance between the smart meter and the $A P_{i}$, and $\mu(x)$ is the Gaussian distribution with the parameter $(0, \sigma)$.

\section{B. Transmission rate}

The transmission rate is an important metric affecting the QoS of data services. It is usually represented by the link reachability rate. Then the link transmission rate of the $A P_{i}$ currently connected to the smart meter is:

$$
C_{i}=W \log _{2}\left(1+S N R_{i}\right) \quad i=1, \cdots, I
$$

where $W$ is the bandwidth and $S N R_{i}$ is the signal to noise ratio.

\section{Load balancing}

The load here includes the bandwidth resource load of the $A P_{i}$ and the computing resource load of the MEC server which is connected to $A P_{i}$. It is defined as the weighted sum of the ratio of the occupied network bandwidth to the total available bandwidth and the ratio of the allocated computing resources of the MEC server to the total available computing resources. It is expressed as:

$$
L_{i}=\lambda_{n}^{B} \frac{\sum_{n \in U_{i}} B_{n i} x_{n i}}{B_{t o t, i}}+\lambda_{n}^{f} \frac{\sum_{n \in U_{i}} f_{n i} x_{n i}}{f_{\text {tot }, i}}
$$

The first part is the bandwidth resource occupancy rate and the second part is the computing resource usage rate, $B_{t o t, i}$ is the total network bandwidth of $A P_{i}, U_{i}$ is the set of all the smart meters connected to $A P_{i}, B_{n i}$ is the service bandwidth requested by the $n$th smart meter in the set, $f_{n i}$ is the computing resources required by the $n$th smart meter in the set to complete the computing task, and $f_{\text {tot }, i}$ is the total computing resources provided by the MEC server that is connected to $A P_{i}$. Moreover, $x_{n i} \in\{0,1\}$, indicating the access indicator of the smart meter $n$, if the smart meter accesses the $A P_{i}$, then $x_{n i}=1$, otherwise, $x_{n i}=0$. And $\lambda_{n}^{B}, \lambda_{n}^{f}$ are the weights.

For the $A P_{i}$ currently connected to the smart meter $n, i \in I=\{1, \ldots, I\}$, and $I$ is the set of all APs in the network, $R S S_{i}$ is the current signal strength of current $A P_{i}$ received by the smart meter $n, R S S_{i} \in \boldsymbol{R}$, and $\boldsymbol{R}$ is the received signal strength range of the smart meter, $D_{R S S_{i}}$ is the change rate of the RSS for the smart meter $n, C_{i}$ is the transmission rate of $A P_{i}$ currently connected to the smart meter, $C_{i} \in \boldsymbol{C}$, and $\boldsymbol{C}$ is the range of the transmission rate, $D_{c_{i}}$ is the change rate of the transmission rate, $L_{i}$ is the current AP load, $L_{i} \in \boldsymbol{L}$, and $\boldsymbol{L}$ is the range of the $A P_{i}$ load, $T_{n i}$ is the time when the data is transmitted from the smart meter $n$ to the current $A P_{i}$. 
The above various information of $A P_{i}$ received by the smart meter is recorded as the state $s_{i}$ of the smart meter relative to $A P_{i}$. It's a quintuple of $\left\{D_{R S S_{n}}, D_{C_{i}}, D_{L_{i}}, D_{d}, T_{n i}\right\}, s \in S$, and $S$ is the set of $s_{i}$. Then:

$$
\boldsymbol{S}=\left\{s_{1}, \cdots, s_{i}, \cdots, s_{I}\right\} \quad i=1,2, \cdots, I
$$

In Equation (5), $S$ is the data set required by the algorithm in this paper. When the smart meter needs to perform offloading, the WKNN-based offloading decision algorithm could be used according to the current state information, to decide on the optimal offloading results.

\subsection{OFFLOADING DECISION BASED ON WKNN}

In general, the location of the AP and MEC servers in the PIoT will not change. Therefore, every successful offloading experience of smart meters can be used as a reference for other smart meters in the future. In this way, each offloading process can be seen as an instance-based machine learning system. Given this, the paper proposes an offloading decision method based on WKNN. This method does not estimate the objective function in the entire instance space at one time but makes local and different estimations for each new instance to be classified.

According to this algorithm, firstly the distance between the samples to be classified $s_{u}$, and each sample in the training set $\boldsymbol{S}$ was calculated using the distance metric function. Afterward, the calculated distance was sorted, to select the $K$ training samples nearest to the sample to be classified as $K$ nearest neighbors of $s_{u}$. Then multiplying the $K$ nearest neighbors by their corresponding weighting coefficients and averaging them, the results were obtained as the final offloading decision results.

\subsubsection{DISTANCE METRIC FUNCTION}

$I_{t}$ indicates the set of target offloaded APs for the smart meter, including current $A P_{i}$ and the number of neighboring APs. Then, the state $s_{u}$ of the smart meter relative to each AP in the set was obtained, as an unknown sample, and the corresponding offloading results of the unknown sample $s_{u}$ were obtained according to the known sample set $\boldsymbol{S}$. The Euclidean distance $d\left(s_{u}, s_{i}\right)$ in the unknown sample and the known sample set was defined as the distance metric function, also called the 'similarity measure', meaning the smaller the distance, the greater the similarity.

$$
d\left(s_{u}, s_{i}\right)=\left\|s_{u}-s_{i}\right\|_{2}=\sqrt{\left|s_{u}-s_{i}\right|^{2}}
$$

According to the above formula, the offloading result of the $K$ samples with the smallest distance from the unknown sample $s_{u}$ is that with the largest similarity with the unknown sample.

\subsubsection{DISTANCE WEIGHTED FUNCTION}

In the actual scene, due to some special reasons, the prior offloading experience will be invalid, and the isolated points will be generated in the offloading knowledge base. These isolated points affect the decision result. Therefore, the algorithm proposed in this paper requires weighting the function of distance, so that the influence of a few isolated points on the judgment will be diluted by the majority of normal data. The distance weighted function used 
in this paper is a Gaussian function that makes the points closer to each other get a larger weight. The distance weighted function is:

$$
w=a e^{-\frac{(d-b)^{2}}{2 c^{2}}}
$$

where, $a, b, c \in R$. Therefore, after sorting the calculated distances, the selected $K$ samples were weighted and averaged, namely:

$$
f(d)=\frac{\sum_{i=1}^{\left|\boldsymbol{I}_{t}\right|} d\left(s_{u}, s_{i}\right) w_{i}}{\sum_{i=1}^{\left|\boldsymbol{I}_{t}\right|} w_{i}}
$$

Then, $f(d)$ is the final offloading decision result.

The specific steps of the algorithm are as follows.

Step1 : The MEC server collects information such as the RSS, transmission rate, AP load, and packet transmission time for $I$ APs, and updates the state $s$ in real-time to obtain a known sample set $\boldsymbol{S}$. $\boldsymbol{S}=\left\{s_{1}, \ldots, s_{I}\right\}$;

Step2: If (the current calculation task of smart meter $n$ is too large to be processed locally)

Then: Calculate the current movement state of the smart meter according to Eq. (1)-(4), denoted as $s_{u}$, calculate the Euclidean distance $d\left(s_{u}, s_{i}\right)$ between the unknown sample $s_{u}$ and the known sample set $\boldsymbol{S}$ according to Eq. (5), and sort these distances from the small to large.

Step3: Set the value of $K$;

Step4: Calculate the weight of the first $K$ distances after sorting according to formula (6);

Step5: Using the results obtained in the previous step, the selected $K$ samples are weighted and averaged according to Eq. (8), to obtain the final offloading result $f(d)$;

Step6: The MEC server transmits the result to the smart meter, and the smart meter performs the offloading operation.

It can be seen from the above that the WKNN-based unloading decision algorithm reasonably examines the multiple attributes of the AP and the MEC server, ensures the load balancing of the system. In addition, the smart meter only needs to propose the offloading request of the computing task according to their own requirements, without the need to consider the hardware and resource information at the MEC server.

\section{ANALYSIS OF SIMULATION RESULTS}

\subsection{SIMULATION SCENES AND PARAMETERS}

In this section, the proposed WKNN-based offloading decision algorithm was verified by computer simulation. Based on the above theoretical model, the traditional unweighted $\mathrm{K}$ nearest neighbor (KNN) offloading decision algorithm and our algorithm were compared and 
analysed. Figure 2 shows the simulation scenario, in which each smart meter was connected to four APs, and the number of MEC servers was four. It's assumed that the coverage of the AP connected to each MEC server is a circular area with a radius of $1000 \mathrm{~m}$, where smart meters are randomly distributed. Detailed simulation parameters are shown in Table 1.
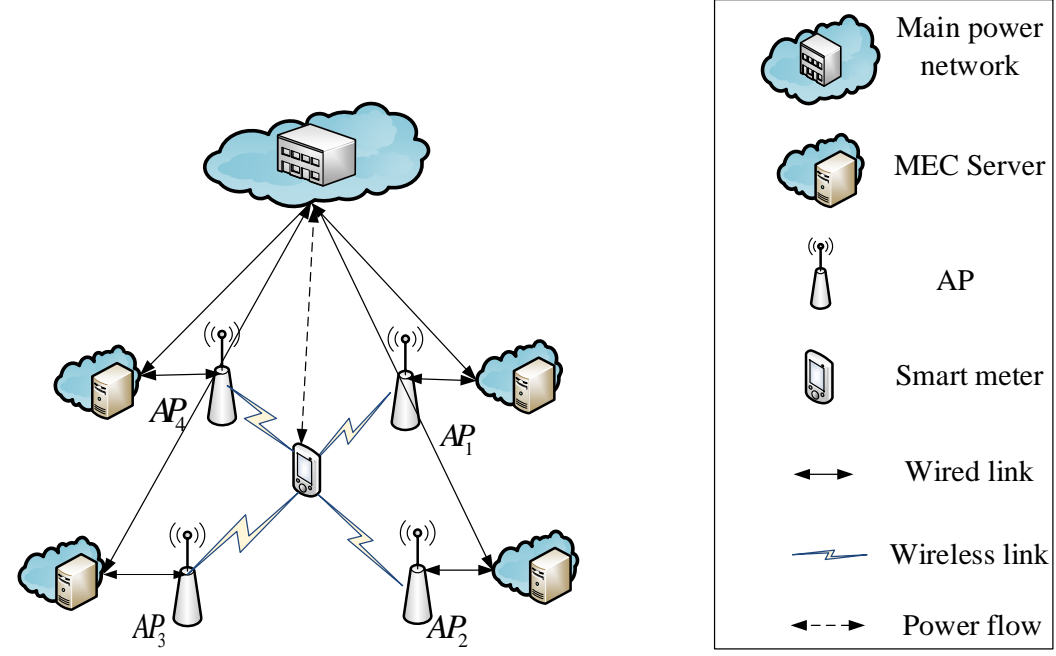

Fig. 2 Simulation scenario

Table 1 System simulation parameters

\begin{tabular}{|c|c|}
\hline Parameter description & Values \\
\hline Channel bandwidth & $5 \mathrm{MHz}$ \\
\hline Signal transmission power $K_{1}$ & $20 \mathrm{dBm}$ \\
\hline Path loss $K_{2}$ & $-100 \mathrm{dBm}$ \\
\hline Computing power of $M E C$ server & $5 \mathrm{GHz} / \mathrm{sec}$ \\
\hline
\end{tabular}

\subsection{RESOURCE UTILIZATION RATE}

In the MEC-based power IoT system scenario, the MEC server is distributed around the smart meter. When offloading the computing tasks on the smart meter, it is necessary to ensure maximizing the resource utilization of each MEC server and achieving the load balancing of the network.

In the simulation experiment, by setting the value range of $K$ to $[1,20]$ and the number of smart meters to [1, 170], we obtained the change of resource utilization when $K$ changes. Figure 3 shows that with the increase of the $K$ value, the resource utilization rate of the offload decision algorithm proposed in this paper becomes larger and is greater than that of the traditional KNN offload decision algorithm. Then $K=19$, the simulation was conducted to obtain the relationship between resource utilization and the number of smart meters, as shown in Figure 4. It can be seen that as the number of smart meters increases, the resource utilization rate gradually increases. The WKNN offload decision algorithm in this paper comprehensively considers the load balancing and transmission rate of each AP and the received signal strength, and weights the average to ensure that each node can be effectively utilized and avoids resource waste. Therefore, its resource utilization is greater than that of the traditional 
algorithm, especially when the number of smart meters is large, making their difference more significant.

\subsection{OFFLOADING DELAY}

The offloading delay of the WKNN-based offloading decision algorithm is compared to the traditional KNN offloading decision algorithm. Figure 5 shows how the offloading delay changes with the $K$ value, our algorithm greatly reduces the offloading delay with a small value of $K$, which is $73 \%$ lower than the traditional KNN offloading decision algorithm. Moreover, at $K=10$, the offloading delay is minimized. Thus, by setting $K=10$ and changing the number of smart meters in the simulation scenarios, we obtain the change of the offloading delay as the number of smart meters increases, as shown in Figure 6. It can be seen from the figure that the offloading delay of our algorithm is reduced by $21 \%$ compared to the traditional KNN offloading decision algorithm.

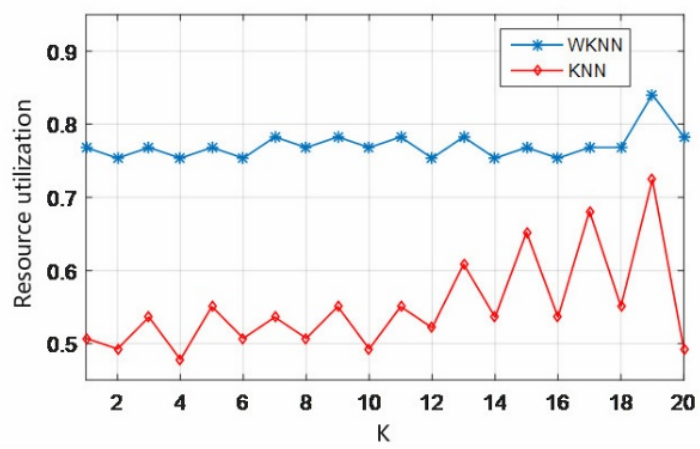

Fig. 3 Comparison of resource utilization with different $K$ values

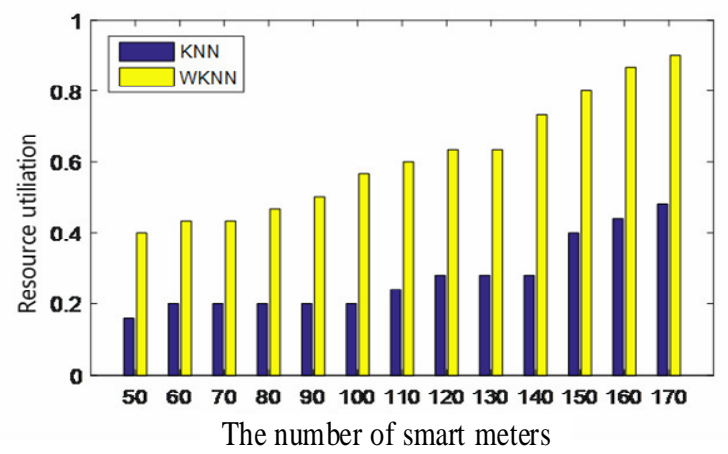

Fig. 4 Comparison of resource utilization with different number of smart meters 


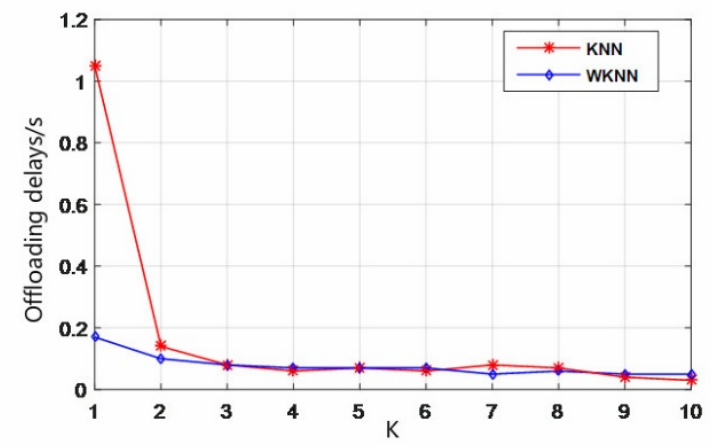

Fig. 5 Comparison of offloading delays with different $K$ values

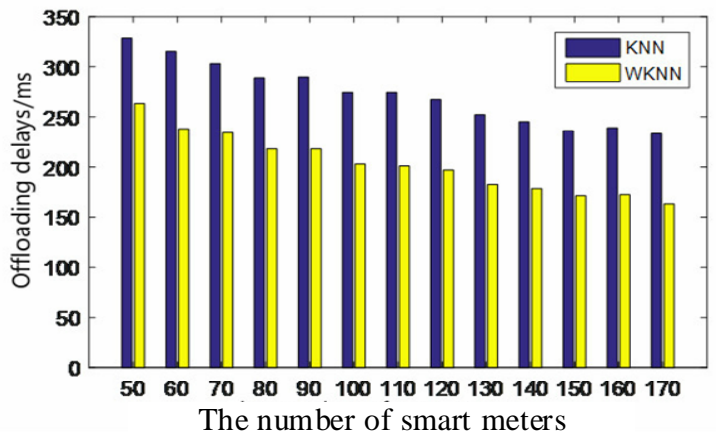

Fig. 6 Comparison of offloading delays with different number of smart meters

\section{CONCLUSIONS}

With the more extensive application of PIoT in smart grids, the data collected from smart meters is exploding, making the computational task of power data more and more complex. Therefore, in order to improve computing power and maximize resource utilization, this paper proposes a WKNN-based offloading decision algorithm. Firstly, the training set required by the WKNN algorithm was collected, including the received signal strength, transmission rate, load balance of the AP and MEC server. Then, using the Gaussian function, a weighted analysis for the Euclidean distance in the sample and the training set was performed. $K$ similar results were finally obtained in the training set, and the results with the largest similarity are the final offloading result. The simulation results showed that the proposed algorithm effectively reduces the offloading delay of computing tasks, and improves the resource utilization when the number of meters increases, ensuring that every MEC server resources around the smart meter can be effectively utilized. In future research, the energy consumption of the MEC server at the time of offloading should be optimized to further improve the overall offloading performance.

\section{ACKNOWLEDGMENTS}

Funded by National Natural Science Foundation of China (No.61741102). 
J. Jia, J. Xu, W. Cui, F. Tao, G. Zhang, C. Hu, Z. Zhang, Z. Liu: Offloading Decision Algorithm Based on Distance Weighted K-Nearest

\section{REFERENCE}

[1] L. Atzori, A. Iera, G. Morabito, The Internet of Things: A survey, Computer Networks, Vol. 54, No. 15, pp. 2787-2805, 2010. https://doi.org/10.1016/j.comnet.2010.05.010

[2] Z.Z. Li, J.X. Liu, Application of mobile edge computing in Internet of Vehicles, Modern Science \& Technology of Telecommunications, Vol. 47, No. 3, pp. 37-41, 2017.

[3] ETSI. GS MEC-IEG 004 V1.1.1 -2015-11. Mobile Edge Computing (MEC); Service Scenarios, 2015.

[4] X.F. He, J. Liu, R.C. Jin, H.Y. Dai, Privacy-Aware Offloading in Mobile-Edge Computing, 2017 IEEE Global Communications Conference (GLOBECOM), No. 12, 2017.

https://doi.org/10.1109/GLOCOM.2017.8253985

[5] L.X. Chen, J. Xu, S. Zhou, Computation Peer Offloading in Mobile Edge Computing with Energy Budgets, 2017 IEEE Global Communications Conference (GLOBECOM), No. 12, 2017. https://doi.org/10.1109/GLOCOM.2017.8255052

[6] J.K. Ren, G.D. Yu, Y.L. Cai, Y.H. He, F.Z. Qu, Partial Offloading for Latency Minimization in Mobile-Edge Computing, 2017 IEEE Global Communications Conference (GLOBECOM), No. 12, 2017. https://doi.org/10.1109/GLOCOM.2017.8254550

[7] D.V. Le, C.K. Tham, An Optimization-based Approach to Offloading in Ad-Hoc Mobile Clouds, 2017 IEEE Global Communications Conference (GLOBECOM), 2017.

https://doi.org/10.1109/GLOCOM.2017.8254632

[8] X.Y. Tao, K. Ota, M.X. Dong, H. Qi, K.Q. Li, Performance Guaranteed Computation Offloading for Mobile-Edge Cloud Computing, IEEE Wireless Communication Letters, Vol. 6, No. 6, pp. 774-777, 2017. https://doi.org/10.1109/LWC.2017.2740927

[9] W. Fan, Y. Liu, B. Tang, F. Wu, Z. Wang, Computation Offloading Based on Cooperations of Mobile Edge Computing-Enabled Base Stations, IEEE Access, Vol. 6, pp. 22622-22633, 2017. https://doi.org/10.1109/ACCESS.2017.2787737

[10] Y. Zhang, K. Liang, S. Zhang, Y.S. He, Applications of edge computing in PIoT, Energy Internet and Energy System Integration (EI2), 2017 IEEE Conference on, IEEE, pp. 1-4, 2017. https://doi.org/10.1109/EI2.2017.8245749

[11] Y. Huang, Y. Lu, F. Wang, X.Y. Fan, J.C. Liu, V.C.M. Leung, An Edge Computing Framework for Real-Time Monitoring in Smart Grid, 2018 IEEE International Conference on Industrial Internet (ICII), IEEE, pp. 99-108, 2018.

https://doi.org/10.1109/ICII.2018.00019

[12] D.H. FU, D. Liu, G.J. Shi, A.X. Wang, C.X. Fan, H.J. Wang, Y.Y. Zhang, Off-line emergency communication method of power internet of things based on edge computing, Telecommunications Science, Vol. 34, No. 3, pp. 183-191, 2018.

[13] B. Li, B.C. Jia, S.S. Chen, B. Yang, Y. Sun, B. Qi, Prospect of Application of Edge Computing in the Field of Supply and Demand, Electric Power, Vol. 51, No. 11, pp. 154-162, 2018.

[14] C. Wang, C. Liang, F.R. Yu, Q.B. Chen, L. Tang, Computation offloading and resource allocation in wireless cellular networks with mobile edge computing, IEEE Transactions on Wireless Communications, Vol. 16, No. 8, pp. 4924-493, 2017.

https://doi.org/10.1109/TWC.2017.2703901 